\title{
DEGENERACY THEOREMS FOR HOLOMORPHIC MAPPINGS BETWEEN ALGEBRAIC VARIETIES
}

BY

\author{
ROBERT MOLZON
}

\begin{abstract}
Degeneracy theorems are proved for holomorphic mappings from affine algebraic manifolds to projective algebraic manifolds of equal dimensions. A mapping is degenerate if it satisfies a growth estimate and omits a set of $k$-plane sections of positive capacity; the capacity being defined in terms of a singular integral. The capacity is a more delicate method of measuring the size of a set of $k$-plane sections than Hausdorff measure and arises naturally by considering the singular integrals in the First Main Theorem of Nevanlinna.
\end{abstract}

0. Introduction. In [1] Bott and Chern give a condition under which a holomorphic mapping $f: C^{n} \rightarrow C^{n}$ must be degenerate. Using the notation of Griffiths and King this condition may be stated as

$$
\lim _{r \rightarrow \infty} t_{n-1}(r) / T_{n}(r)=0 \text { and } f \text { omits an open set. }
$$

This condition implies that the image of $f$ does not contain an open set. The terms $t_{n-1}(r)$ and $T_{n}(r)$ are the Nevanlinna order functions of $f$ which measure certain growth properties of $f$.

One may contrast the above result with the following well-known extension of the classical Picard theorem: If $f: C^{n} \rightarrow \mathbf{P}^{n}$ omits $n+2$ hyperplanes in general position then $f$ is degenerate.

In a previous paper [8] the author studied holomorphic mappings $f: C^{n} \rightarrow C^{n}$ and showed that if $f$ satisfied $\lim _{r \rightarrow \infty} t_{n-1}(r) / T_{n}(r)=0$ and is nondegenerate then $f$ omits at most a set of Hausdorff dimension $2 n-2$.

In this paper I consider holomorphic mappings $f: A \rightarrow M$ where $A$ is an affine algebraic manifold and $M$ is a projective algebraic manifold, $A$ and $M$ having the same dimension. Degeneracy theorems are then proved for $f$ satisfying a condition which is, loosely stated

$$
\begin{aligned}
& \lim _{r \rightarrow \infty} t_{q-1}(r) / T_{q}(r)=0 \text { and } f \text { omits a set } E, \text { of codimension } q \\
& \text { linear spaces, of Hausdorf dimension }>k(n, q) .
\end{aligned}
$$

Here $k(n, q)$ is a constant depending upon $n$ and $q$.

These degeneracy theorems assume $f$ omits a set which is quite small; in fact if Hausdorff dimension is used to measure the "size" of a set, these degeneracy theorems are the best possible.

Received by the editors May 12, 1980 and, in revised form, December 9, 1980.

1980 Mathematics Subject Classification. Primary 32H25, 32A15, 32J25.

(C) 1982 American Mathematical Society 0002-9947/81/0000-1030/\$03.50 
1. Residue formulae. The starting point in our discussion is a residue formula which generalizes the classical Poincaré equation. A complete discussion is given in [6].

Let $Z$ denote a point in $C^{n}$. We will use the notation

$$
\theta_{l}=\left(d d^{c} \log |Z|^{2}\right), \quad \Theta_{l}=\log |Z|^{2} \theta_{l} .
$$

We then have the following:

Proposition 1.1 (SEe [6]). Let $U$ be an n-dimensional complex manifold and $F$ : $U \rightarrow C^{n}$ a holomorphic map. The forms $F^{*} \theta_{l}$ and $F^{*} \Theta_{l}$ are locally integrable for all $l$, and if $W=F^{-1}(0)$ has pure codimmension $r$, then $d d^{c} F^{*} \Theta_{l-1}=\theta_{l}$ for $l<r$ and $d d^{c} F_{r-1}^{*}=W$ in the sense of distributions.

A global version of this is given on projective space $P^{n} C$ by the following construction.

Let $e_{1}(Z), \ldots, e_{d}(Z)$ be hyperplane sections and $D_{1}, \ldots, D_{d}$ the corresponding divisors on $P^{n} C$. Assume $L=D_{1} \cdots D_{d}$ is a linear space of codimension $d$. Let $\omega$ be the Kähler form of the Fubini-Study metric on $P^{n}$ and let

$$
\begin{aligned}
& \omega_{0}=\omega+d d^{c} \log \|e(z)\|^{2}, \\
& \Lambda=\log \left(\frac{1}{\|e(z)\|^{2}}\right) \sum_{k=0, d-1} \omega_{0}^{d-1-k} \wedge \omega^{k}, \\
& e(z)=e_{1}(z) \wedge \cdots \wedge e_{d}(z) .
\end{aligned}
$$

PROPOSITION 1.2 (SEE [6]). Under the above assumption that $L=D_{1} \cdots \cdot D_{d}$ has pure codimension $d$, one has the equation of currents

$$
d d^{c} \Lambda=\omega^{d}-[L] .
$$

Proof (SKetch). Let $\left\{\zeta_{i}\right\}$ be local coordinates on $P^{n}$ such that $D_{i}=\left\{\zeta_{i}=0\right\}$, $i=1, \ldots, d$. Hence $e_{i}(\zeta)=\zeta_{i}, i=1, \ldots, d$. Then $\omega_{0}^{l}=\zeta^{*} \theta_{l}$ and

$$
d d^{c} \Lambda=\sum_{k=0}^{d-1}\left[\zeta^{*} \theta_{d-1-k} \wedge \omega^{k+1}-d d^{c}\left(\zeta^{*} \Theta_{d-1-k} \wedge \omega^{k}\right)\right] .
$$

By Proposition 1.1 this series telescopes to give

$$
d d^{c} \Lambda=\omega^{d}-D_{1} \cdots \cdot D_{d} .
$$

Now suppose $M$ is a smooth projective algebraic variety. Let $H$ be the hyperplane section bundle over $M$. Let $V$ be the vector space of holomorphic sections $\Gamma(M, H)$ with basis $\left\{e_{0}, \ldots, e_{N}\right\}$. Denote by $G_{q}(V)$ the Grassmannian of codimension $q$ linear spaces of $P(V)$. Given a hermitian metric on $V$ we obtain in the usual way a Fubini-Study Kähler metric on $G_{q}(V)$. Denote the corresponding exterior form by $\omega$.

We may now give the residue theorem on the Grassmannian.

COROllaRY 1.3. Let $a \in G_{q}(V)$. There exists a form $\Omega_{a}$ on $G_{q}(V)$ such that

$$
d d^{c} \Omega_{a}=\omega^{d}-\delta_{a} ; \quad d=\operatorname{dim} G_{q}(V)=q(N-q+1) .
$$


Proof. This result is contained in Hirschfelder's Theorem 4.2 of [7]. It is important that we be able to determine the singularity of $\Omega_{a}$. This may be done in the following manner. Embed $G_{q}(V)$ in $\mathbf{P}^{K}(V)$ by the Plücker embedding. Then $a \in G_{q}(V) \subset \mathbf{P}^{K}(V)$. Choose $d$ hyperplanes of $\mathbf{P}^{K}(V)$ such that in a neighborhood $U \subset G_{q}(V)$ of $a$ we have $a=H_{1} \cdots \cdot H_{d}$. Let $\Lambda$ be the singular form on $\mathbf{P}^{K}(V)$ associated to $L=H_{1} \cdots \cdot H_{d}$ as in Proposition 1.2. The restriction of $\Lambda$ to $U \subset G_{q}(V)$ will then have the same singularity as $\Omega_{a}$.

Let $M$ be a smooth projective algebraic variety as above. Suppose $L$ is a subvariety of $M$ of pure codimension $q$ given by $L=D_{1} \cdots \cdot D_{q}$, with $D_{i}$ the divisor of $\sigma_{i}$, a holomorphic section, $\sigma_{i} \in \Gamma(M, H)$. We then have

COROLlaRY 1.4. There exists a form $\Lambda$ on $M$ such that

$$
d d^{c} \Lambda=\omega^{q}-[L] \text {. }
$$

Here $\omega$ denotes the Kähler form of the Fubini-Study metric on M.

Proof. First define the variety $X \subset M \times G_{q}(V)$ by $X=\{(x, Y) \mid x \in L(Y)\}$. $L(Y)$ denotes the subvariety of $M$ determined by the intersection of divisors $D_{1}, \ldots, D_{q}$ defining $L \in G_{q}(V)$. We then have the projections: $\pi: X \rightarrow M$, $\rho: X \rightarrow G_{q}(V)$. Let $a \in G_{q}(V)$ : define $L=L(a)$ as in the statement of the corollary. Let $\Omega_{a}$ denote the form on $G_{q}(V)$ satisfying

$$
d d^{c} \Omega_{a}=\omega_{1}^{d}-\delta_{a} .
$$

$\omega_{1}$ is the Kähler form on $G_{q}(V)$ of the Fubini-Study metric. Define $\Lambda=\pi_{*} \rho^{*} \Omega_{a}$. We then claim $d d^{c} \Lambda=\omega^{q}-[L]$. First of all we remark that $\pi_{*}$ is defined by duality; that is, if $\psi$ is a current then $\left(\pi_{*} \psi, \phi\right)=\left(\psi, \pi^{*} \phi\right)$ where $\phi$ is a smooth form with compact support (and with appropriate degree). One then checks that $\pi_{*} \rho^{*} \delta_{a}$ $=L(a)$ by approximating $\delta_{a}$ with approximate identities. Since $\pi_{*}$ is defined by duality it lowers bidegree of a current by $\operatorname{dim} \pi^{-1}(x)=q(N-q)$. Therefore

$$
\text { bideg } \pi_{*} \rho^{*} \omega_{1}^{d}=q(N-q+1)-q(N-q)=q \text {. }
$$

In fact one has $\pi_{*} \rho^{*} \omega_{1}^{d-i}=\omega^{q-i}$. Computing $\pi_{*} \rho^{*} \Omega_{a}$ one obtains

$$
\pi_{*} \rho^{*} \Omega_{a}=\log \left(\frac{1}{\|\sigma(x)\|^{2}}\right)_{k=0}^{q-1} \omega_{0}^{q-1-k} \wedge \omega^{k},
$$

where $\omega$ is the Kähler form of the Fubini-Study metric, $\omega_{0}=d d^{c} \log \|\sigma(x)\|^{2}+\omega$ and $\|\sigma\|^{2}=\left|\sigma_{1}(x)\right|^{2}+\cdots+\left|\sigma_{q}(x)\right|^{2}, \sigma_{i}$ being holomorphic sections of the hyperplane section bundle. Hence $\pi_{*} \rho^{*} \Omega_{a}$ is the Levine form of [6].

It will be important to have an explicit expression for the singularity of the form $\Omega_{a}$ on $G_{q}(V)$. We use the results of Hirschfelder [7]. Choose holomorphic coordinates $\left\{\zeta^{1}, \ldots, \zeta^{d}\right\}$ on $G_{q}(V)$ such that $a \sim \zeta^{\prime}=\zeta^{d}=0$. Writing the Levine form in the coordinates $\zeta$ we have

$$
\Omega_{a}(\zeta)=\log \frac{1}{|\zeta|^{2}} \cdot \frac{1}{|\zeta|^{2 d-2}} \cdot \Phi(\zeta),
$$

where $\Phi(\zeta)$ is a nonsingular $(d-1, d-1)$ form unformly bounded in $\zeta$. 
Now suppose $\mathbf{b} \in G_{q}(V)$ is a point in the $\zeta$-coordinate neighborhood of $a$. We need an expression for $\Omega_{\mathrm{b}}(\zeta)$. Choose holomorphic coordinates $\zeta^{\prime}$ on $G_{q}(V)$ such that $\mathbf{b} \sim \zeta_{1}^{\prime}=\cdots=\zeta_{d}^{\prime}=0$. Then $\Omega_{\mathbf{b}}(\zeta)=\log 1 /\left|\zeta^{\prime}\right| \cdot\left[1 /\left|\zeta^{\prime}\right|^{2(d-1)}\right] \Phi^{\prime}\left(\zeta^{\prime}\right)$. Writing $\zeta^{\prime}$ in terms of $\zeta$ we have

$$
\Omega_{\mathrm{b}}(\zeta)=\log 1 /|\zeta-b|^{2} \cdot\left[1 /|\zeta-b|^{2(d-1)}\right] \tilde{\Phi}(\zeta),
$$

where on the right-hand side of the above equation we use $b$ to denote the $\zeta$-coordinates of the point $\mathbf{b}$. Here $\tilde{\Phi}(\zeta)$ is a $(d-1, d-1)$ form which is uniformly bounded in $\zeta$ and $b$. It follows that we have the inequality

$$
\Omega_{\mathrm{b}}(\zeta) \leqslant \frac{1}{|\zeta-b|^{2(d-1)+\varepsilon}} C_{e} \omega^{d-1}(\zeta),
$$

where $C_{\varepsilon}$ is a constant depending only upon $\varepsilon>0$ and $\omega$ is the Kähler form of the Fubini-Study metric on $G_{q}(V)$.

2. The First Main Theorem (FMT). In $\S 1$ we wrote down some residue theorems. These may be integrated after pulling back the currents under holomorphic mappings to give various forms of the FMT.

Let $A$ be a smooth affine algebraic variety of dimension $n$. Let $\tau: A \rightarrow[-\infty, \infty)$ be a special exhaustion function on $A$; that is, $\tau$ has only finitely many logarithmic singularities, finitely many critical values, and the Levine form $d d^{c} \tau$ satisfies:

(a) $d d^{c} \tau \geqslant 0$,

(b) $\left(d d^{c} \tau\right)^{n-1} \not \equiv 0$ on $T_{z}^{0,1}(b A[r])$,

(c) $\left(d d^{c} \tau\right)^{n} \equiv 0$.

Here $A[r]=\{x \in A \mid \tau(x) \leqslant r\}$ and $b A[r]=\{x \in A \mid \tau(x)=r\}$. Such a $\tau$ exists by Proposition 2.4 of [6].

Let $M$ be a smooth projective algebraic variety of dimension $n$. Let $H$ denote the hyperplane section bundle over $M$ and let $V=\Gamma(M, H)$ be the vector space of holomorphic sections of $H$.

Let $G_{q}(V)$ be the Grassmannian of codimension $q$ linear subspaces of $P(V)$. Let $Z=D_{\sigma_{1}} \cdots \cdot D_{\sigma_{q}}$ where $D_{\sigma_{i}}$ is the divisor of $\sigma_{i} \in V$. Assume $Z$ has pure codimension $q$. We must introduce some notation. First let $\Lambda$ denote the singular $(q-1, q-1)$ form of Corollary 1.4. Let $\psi=d d^{c} \tau, \tau$ being the special exhaustion function on $A$. Suppose $f: A \rightarrow M$ is a holomorphic mapping. Put $Z_{f}[t]=$ $A[t] \cap f^{-1}(Z)$. The following terms then appear in the FMT:

$$
\begin{gathered}
N(Z, r)=\int_{0}^{r}\left\{\int_{Z_{f}[t]} \psi^{n-q}\right\} d \log t, \\
t_{q}(r)=\int_{A[r]}\left(f^{*} \omega\right)^{q} \wedge \psi^{n-q}, \\
T_{q}(r)=\int_{0}^{r} t_{q}(s) d \log s, \\
m_{q}(Z, r)=\int_{b A[r]} f^{*} \Lambda \wedge d^{c} \tau \wedge \psi^{n-q}, \\
S_{q}(Z, r)=\int_{A[r]} f^{*} \Lambda \wedge \psi^{n-q+1} .
\end{gathered}
$$


We assume that $f^{-1}(Z)$ does not pass through any of the logarithmic singularities of $\tau$ in the above definitions. If $f^{-1}(Z)$ passes through logarithmic singularities of $\tau$, the counting function $N(Z, r)$ may be modified by using the Lelong number of $Z$ at the singularity.

We may now state the FMT.

Proposition 2.1 (FMT). Using the above notation with $f: A \rightarrow M$ a nondegenerate holomorphic mapping one has

$$
N(Z, r)+m_{q}(Z, r)=T_{q}(r)+S_{q}(Z, r)+O(1),
$$

where $\theta(1)$ is a constant depending upon $Z$ but not on $r$.

ReMark. The proof of the FMT follows from a double integration of the pull back by $f^{*}$ of the residue formula stated in Corollary 1.4 .

COROLlary 2.2. With the above notation and the assumption that $f^{-1}(Z)$ does not meet a logarithmic singularity of $\tau$ one has the inequality

$$
T_{q}(r) \leqslant N(Z, r)+m_{q}(Z, r)+c,
$$

where $c$ is a constant (does not depend $r$ on $Z$ ).

Proof. Choose $r_{0}$ so large that $A\left[r_{0}\right]$ contains all of the logarithmic singularities of $\tau$. The constant term (1) may then be expressed as

$$
\vartheta(1)=N\left(Z, r_{0}\right)+m\left(Z, r_{0}\right)-S_{q}\left(Z, r_{0}\right)-T_{q}\left(r_{0}\right) .
$$

The right-hand side of (2.1) then becomes

$$
T_{n}(r)-T_{n}\left(r_{0}\right)+S_{q}(Z, r)-S_{q}\left(Z, r_{0}\right)+N\left(Z, r_{0}\right)+m\left(Z, r_{0}\right) \text {. }
$$

Now the Levine form $\Lambda$ is positive on $M$. Since $f^{*}$ preserves positivity we have $S_{q}(Z, r)-S_{q}\left(Z, r_{0}\right) \geqslant 0$. Because $d^{c} \tau \wedge \psi^{n-q}$ is a positive form on $b A[r]$ it also follows that $m\left(Z, r_{0}\right)$ is nonnegative. Since $T_{q}\left(r_{0}\right)$ is a constant independent of $Z$ and $r$ the result follows.

3. Capacity. The tool from analysis which allows us to obtain good estimates on the size of an omitted set of a holomorphic mapping is Newtonian capacity. We want to extend the idea of capacity from Euclidean space to a manifold in a way which allows us to easily see the geometric implications.

We begin by recalling the definitions on $R^{n}$. The Newtonian kernel $K_{\alpha}$ is given by

$$
K_{\alpha}(x, y)=1 /|x-y|^{\alpha} .
$$

Let $\mu$ be a positive Borel measure with compact support on $E \subset R^{n}$. The $\alpha$ potential of $\mu$ is given by

$$
V_{\mu}(x)=\int_{R^{n}} K_{\alpha}(x, y) d \mu(y) .
$$

The energy of the measure $\mu$ is given by

$$
\varepsilon_{\mu}=\int_{R^{n}} V_{\mu}(x) d \mu(x) .
$$

We have the following basic result. 
Proposition 3.1. Given a compact Borel set $E \subset R^{n}$ and a charge $Q>0$ there exists a measure $\mu$, called an equilibrium measure, supported on $E$ such that $\mathcal{E}_{\mu}$ is a minimum.

Put $V=\max _{x \in E} V_{\mu}(x)$ for $\mu$ the equilibrium measure on $E$. Provided $V$ exists put $C_{\alpha}(E)=Q / V$. $C_{\alpha}(E)$ is the $\alpha$-capacity of $E$. If $V$ does not exist put $C_{\alpha}(E)=0$ and in this case say $E$ has $\alpha$-capacity zero. Capacity has the properties that: it is subadditive on Borel sets and the $\alpha$-capacity of a point is zero for $\alpha>0$. Thus $\alpha$-capacity is almost a measure.

We want to extend to manifolds the idea of capacity. For our purposes it will only be necessary to define sets of capacity zero and sets of positive capacity. Let $M$ be a complex manifold and $E \subset M$ a compact Borel set.

Definition 3.2. $E \subset M$ has $\alpha$-capacity zero if for every $p \in E$ and every coordinate neighborhood $(U, \phi)$ with $p \in U$, the set $\phi(U \cap E) \subset C^{n}$ has $\alpha$-capacity zero. Similarly we say $E$ has positive $\alpha$-capacity if there exist a point $p \in E$ and a coordinate neighborhood $(U, \phi)$ of $p$ such that $\phi(U \cap E)$ has positive $\alpha$-capacity.

We must show that this definition makes sense. Suppose $(U, \phi)$ is a coordinate neighborhood of $p$ and $\phi(U \cap E)$ has $\alpha$-capacity zero. Let $(\tilde{U}, \tilde{\phi})$ be another coordinate neighborhood of $p$. We claim that the $\alpha$-capacity of $\tilde{\phi}(U \cap \tilde{U} \cap E)$ is zero. Let $F=\phi(U \cap \tilde{U} \cap E)$ and $\tilde{F}=\tilde{\phi}(U \cap \tilde{U} \cap E)$. Let $\psi=\phi \cdot \tilde{\phi}^{-1} ; \psi$ : $\phi(U \cap \tilde{U}) \rightarrow \phi(U \cap \tilde{U})$ with $\psi(\tilde{F})=F$.

We must therefore show that under the "change of variable" $\psi$ sets of capacity zero are mapped to sets of capacity zero. We observe that the $\alpha$-capacity of a compact Borel set $E$ may be obtained as follows. Let $\Gamma_{E}$ be the positive Borel measures supported on $E$ with $V_{\mu}(x) \leqslant 1$ for $x \in E$. Then the $\alpha$-capacity is given by $C_{\alpha}(E)=\sup _{\mu \in \Gamma_{E}} \mu(E)$.

Suppose the set $F$ has positive $\alpha$-capacity. Then there exists a measure $\mu$ on $C^{n}$ supported on $\tilde{F}$ with $V_{\mu}(x) \leqslant 1$ on $\tilde{F}$ and $\mu(\tilde{F})>0$. By the change of variable formula for integration there exists a measure $\nu$ supported on $F$ with $V_{\nu}(y) \leqslant K$ on $F$ and $\nu(F)>0$. Hence by multiplying $\nu$ by $1 / K$ we get a measure $\nu$ with $V_{\nu^{\prime}}(y) \leqslant 1$ on $F$ and $\nu^{\prime}(F)>0$. Therefore $C_{\alpha}(F)>0$. It follows that if $F$ has $\alpha$-capacity zero then $\tilde{F}$ has $\alpha$-capacity zero.

In order to give reasonable geometric interpretation to our results the size of sets will be indicated in terms of Hausdorff dimension. Let $E \subset C^{n}$ be a compact Borel set. Denote by $\mathcal{H}_{\alpha}(E)$ the $\alpha$-Hausdorff measure of $E$. If $\mathcal{H}_{\alpha}(E)=0$ for all $\alpha>k$ and $\mathcal{H}_{\alpha}(E)=\infty$ for all $\alpha<k$, we say $E$ has Hausdorff dimension $k$.

The following relationship between Hausdorff measure and capacity is proved in [2].

Proposition 3.3. If $E \subset C^{n}$ is a compact Borel set then $\mathcal{H}_{\alpha}(E)<\infty$ implies $C_{\alpha}(E)=0$. Conversely, if $C_{\alpha}(E)=0$ then $\mathcal{H}_{\alpha+\varepsilon}(E)=0$ for all $\varepsilon>0$.

In particular, if $C_{\alpha}(E)=0$ then the Hausdorff dimension of $E$ is $\leqslant \alpha$.

Now suppose $M$ is a Kähler manifold. The Hausdorff measure on $M$ is defined in terms of the Kähler metric on $M$. We have the following analogue of Proposition 3.3. 
Proposition 3.4. Let $E \subset M$ be a compact Borel set. Let $\mathcal{H}_{\alpha}$ denote the $\alpha$-Hausdorff measure on $M$. If $\mathcal{H}_{\alpha}(E)<\infty$ then $E$ has $\alpha$-capacity zero in the sense of Definition 3.2. Conversely if $E$ has $\alpha$-capacity zero then $\mathcal{H}_{\alpha+e}(E)=0$ for all $\varepsilon>0$.

Proof. This result follows immediately by pulling everything down to $C^{n}$ by coordinate maps and using Proposition 3.3.

4. Degeneracy theorems. We are interested in finding conditions under which a holomorphic mapping $f: A \rightarrow M$ must be degenerate; that is, the image does not contain an open set. We prove two theorems in this direction.

Let $A$ be a smooth affine algebraic variety and $M$ a smooth projective algebraic variety.

Suppose $M \subset P^{N}$ is given by the sections $\left\{e_{0}, \ldots, e_{N}\right\}$ of $V=\Gamma(M, H)$. Let $\operatorname{dim}_{C} A=\operatorname{dim}_{C} M=n$. Let $f: A \rightarrow M$ be a holomorphic mapping. Let $L(a)$ denote the linear space corresponding to $a \in G_{q}(V)$ where we use the notation of $\$ 1$.

DEFINITION 4.1. The holomorphic map $f$ omits the set $L(a)$ if

$$
\int_{f^{-1}(L(a))[t]} \psi^{n-q}=0 \text { for all } t
$$

DeFINITION 4.2. The holomorphic map $f$ covers $L(a)$ finitely often if

$$
\int_{f^{-1}(L(a))[t]} \psi^{n-q} \leqslant K \text { for all } t .
$$

EXAmPLe. Suppose $f: C^{n} \rightarrow P^{n}$ is a polynomial map. If $L_{q} \subset P^{n}$ is a linear space of codimension $q$ then $\int_{f^{-1}\left(L_{q}\right)[t]} \psi^{n-q}$ is bounded, the exhaustion function on $C^{n}$ being $\tau(z)=\log |z|$.

We now state the main results.

THEOREM 4.3. Let $f: A \rightarrow M$ be a holomorphic mapping. Suppose

$$
\lim _{r \rightarrow \infty} t_{q-1}(r) / T_{q}(r)=0 .
$$

If $f$ omits a set $\left\{L(a) \mid a \in G_{q}(V)\right\}$ of linear spaces of Hausdorff dimension greater than $2 q(N-q+1)-2$ then $f$ is degenerate; that is, the image of $f$ does not contain an open set.

THEOREM 4.4. Let $f: A \rightarrow M$ be a holomorphic mapping and suppose

$$
\lim _{r \rightarrow \infty} \log r / T_{q}(r)=0 \text { and } \lim _{r \rightarrow \infty} t_{q-1}(r) / T_{q}(r)=0 .
$$

If there exists a set $\left\{L(a) \mid a \in G_{q}(V)\right\}$ of linear spaces of Hausdorff dimension greater than $2 q(N-q+1)-2$ which is not covered infinitely often by $f$ then $f$ is degenerate.

Before we prove these two theorems we need two lemmas.

LeMmA 4.5. The proximity term $m_{q}(Z(a), r)$ exists for all $a \in G_{q}(V)$ and is $a$ measurable function of $a$. 
Proof. If $Z(a)$ is a linear space of codimension $q$ such that $f^{-1}(Z(a))$ has codimension $q$ then of course $m(Z(a), r)$ exists. If $f^{-1}(Z(a))$ is of codimension less than $q$ the result follows by blowing up along $Z(a)$. Letting $\hat{M}$ be the blow up of $M$ at $Z(a)$ one computes $\sigma^{*} \Lambda_{Z(a)}$ in local coordinates. Here $\Lambda_{Z(a)}$ is the Levine form giving the residue formula for $Z(a)$. One notes that singularity is sufficiently nice that we may integrate. See [6] or [7] for detailed calculation. Since $\Lambda_{\mathbf{Z ( a )}}$ involves rational functions and logarithms of rational functions it follows that $m(Z(a), r)$ is a measurable function of $a$.

Let $d=\operatorname{dim}_{C} G_{q}(V)=q(N-q+1)$ as before.

Lemma 4.6. Let $E \subset G_{q}(V)$ be a set of finite positive $2(d-1)+\varepsilon$ capacity. Then there is an equilibrium measure $\mu$ supported on $E$ such that $\mu(E)=1$ and

$$
\int_{E} \Omega_{b} d \mu(b) \leqslant C_{e} \omega^{d-1} .
$$

Here $\omega$ is the Kähler form of the Fubini-Study metric on $G_{q}(V)$.

Proof. We may assume $E$ is contained in a coordinate neighborhood $U$ on $G_{q}(V)$, otherwise simply cut off $E$ outside some coordinate neighborhood and define $\mu$ with support on this new set. Let $\zeta$ be the coordinate function on $U$ so

$$
\Omega_{b}(\zeta) \leqslant C_{\varepsilon}\left(|\zeta-b|^{2(d-1)+\varepsilon}\right)^{-1} \cdot \omega^{d-1}(\zeta),
$$

where $C_{\varepsilon}$ is a constant depending only upon $\varepsilon>0$. Let $F=\zeta(E)$. By Definition 3.2 $F$ has positive finite $2(d-1)+\varepsilon$ capacity. Let $\nu$ be the equilibrium measure on $F$ normalized so that $\nu(F)=1$. Now

$$
\begin{aligned}
\int_{F} C_{e} \cdot \frac{1}{|\zeta-b|^{2(d-1)+\varepsilon}} \omega^{d-1}(\zeta) d \nu(b) & =C_{\varepsilon}\left[\int_{F} \frac{1}{|\zeta-b|^{2(d-1)+\varepsilon}} d \nu(b)\right] \omega^{d-1} \\
& \leqslant C_{\varepsilon} V_{\nu}(z) \omega^{d-1}<C_{\varepsilon} \cdot V \omega^{d-1}
\end{aligned}
$$

since $F$ has positive $2(d-1)+\varepsilon$ capacity. Now define $\mu=\nu \circ \phi$ to get the desired measure on $G_{q}(V)$.

Proof of Theorem 4.3. Suppose $f: A \rightarrow M$ omits a set $\left\{L(a) \mid a \in G_{q}(V)\right\}$ of Hausdorff dimension greater than $2 q(N-q+1)-2$. Using the relationship between capacity and Hausdorff measure $f$ omits a set $E$ (in $G_{q}(V)$ ) of positive $2 q(N-q+1)-2+\varepsilon$ capacity for some $\varepsilon>0$. Let $\mu$ be the measure given by Lemma 4.6. Integrate inequality (2.2) with respect to the measure $\mu$. Since $\mu(E)=1$ we have

$$
T_{q}(r) \leqslant \int_{E} N(Z(a), r) d \mu(a)+\int_{E} m_{q}(Z(a), r) d \mu(a)+c .
$$

Since we are assuming $f$ omits $Z(a)$ for $a \in E$ we get

$$
\begin{aligned}
& T_{q}(r) \leqslant \int_{E} m_{q}(Z(a), r) d \mu(a)+c, \\
& T_{q}(r) \leqslant \int_{E} m_{q}(Z(a), r) d \mu(a)+c, \\
& T_{q}(r) \leqslant \int_{E}\left(\int_{b A[r]} f^{*} \Lambda_{a} \wedge d^{c} \tau \wedge \psi^{n-q}\right) d \mu(a)+c .
\end{aligned}
$$


Now

$$
\begin{aligned}
\int_{E}\left(\int_{b A[r]} f^{*} \Lambda_{a} \wedge d^{c} \tau \wedge \psi^{n-q}\right) d \mu(a) \\
=\int_{b A[r]} f^{*}\left[\pi_{*} \rho^{*}\left(\int_{E} \Omega_{a} d \mu(a)\right)\right] \wedge d^{c} \tau \wedge \psi^{n-q} .
\end{aligned}
$$

Applying Lemma 4.6 we get

$$
\begin{aligned}
T_{q}(r) & \leqslant C_{\varepsilon} \int_{b A[r]} f^{*}\left[\pi_{*} \rho^{*} \omega_{1}^{d-1}\right] \wedge d^{c} \tau \wedge \psi^{n-q} \\
& \leqslant C_{\varepsilon} \int_{b A[r]} f^{*}\left(\omega^{q-1}\right) \wedge d^{c} \tau \wedge \psi^{n-q} \leqslant C_{\varepsilon} t_{q-1}(r),
\end{aligned}
$$

where $\omega_{1}$ is the Kähler form on $G_{q}(V)$ and $\omega$ the Kähler form on $M$. We have

$$
T_{q}(r) \leqslant C_{\varepsilon} t_{q-1}(r)+c .
$$

But we have assumed $\lim _{r \rightarrow \infty} t_{q-1}(r) / T_{q}(r)=0$. Since $\lim _{r \rightarrow \infty} 1 / T_{q}(r)=0$ (unless $f$ degenerate) we obtain the result.

Proof of Theorem 4.4. Apply the same argument as above. This time, however, we have the term

$$
\int_{E} N(Z(a), r) d \mu(a)=\int_{E}\left[\int_{0}^{r}\left\{\int_{Z_{f}(a)[t]} \psi^{n-q}\right\} d \log t\right] d \mu(a) .
$$

The assumption of Theorem 4.4 gives

$$
\int_{0}^{r}\left(\int_{Z_{f}(a)[t]} \psi^{n-q}\right) d \log t \leqslant \log r+c
$$

the constant $c$ being the Lelong number (see [6]). The conclusion then follows as in the proof of Theorem 4.3.

5. Example. In the introduction, we remarked that if Hausdorff dimension were used to measure the "size" of an omitted set then the degeneracy theorems above are best possible. By this we mean there exist analytic mappings $f: A \rightarrow M$ which are nondegenerate and satisfy the growth conditions of Theorem 4.3 (or 4.4) yet omit sets of linear spaces in $G_{q}(V)$ of Hausdorff dimmension $2 q(N-q+1)-2$, the extremal dimension of Theorem 4.3. The growth condition $\lim _{r \rightarrow \infty} t_{q-1}(r) / T_{q}(r)=0$ of Theorems 4.3 and 4.4 may be replaced by lim $\inf _{r \rightarrow \infty} t_{q-1}(r) / T_{q}(r)=0$ with no change in the proof. Using this growth condition, we construct an example showing that results 4.3 and 4.4 are best possible.

Let $f: \mathbf{C}^{2} \rightarrow \mathbf{P}^{2}$ be given by $f(z, w)=\left[1: e^{z}: e^{w}\right]$. This map is clearly nondegenerate and omits a set of points (linear spaces of codimension 2) of Hausdorff dimension 2 , namely the projective lines $\left\{\left[w_{0}: w_{1}: w_{2}\right] \mid w_{1}=0\right\}$ and $\left\{\left[w_{0}: w_{1}\right.\right.$ : $\left.\left.w_{2}\right] \mid w_{2}=0\right\}$. In this example $N=2$ and $q=2$; hence $2 q(N-q+1)-2=2$. It remains to show that $\lim \inf _{r \rightarrow \infty} t_{1}(r) / T_{2}(r)=0$. A computation by Carlson and Griffiths [3] gives $T_{1}(r) \leqslant c r$ and $T_{2}(r) \geqslant c^{\prime} r^{2}$. It follows that there exists a 
sequence $r_{\nu} \rightarrow \infty$ such that $t_{1}\left(r_{\nu}\right) \leqslant 2 c \cdot r_{\nu}$ (see [8]). Hence

$$
\liminf _{r \rightarrow \infty} \frac{t_{1}(r)}{T_{2}(r)} \leqslant \lim _{\nu \rightarrow \infty} \frac{t_{1}\left(r_{\nu}\right)}{T_{2}\left(r_{\nu}\right)}, \quad \lim _{\nu \rightarrow \infty} \frac{2 c}{c^{\prime} r_{\nu}}=0 .
$$

We also have

$$
\lim _{r \rightarrow \infty} \log \frac{r}{T_{2}(r)}=0
$$

Thus $f$ satisfies the conditions of Theorem 4.4 and omits a set of points of Hausdorff dimension 2. Thus $2 q(N-q+1)-2$ is the best upper bound for the dimension of a set in $G_{q}(V)$ which may be omitted by a nondegenerate holomorphic mapping satisfying the growth conditions of Theorem 4.4.

Remark. By using the tools of Newtonian capacity and Nevanlinna theory we have been able to obtain results on holomorphic mappings far more delicate than those previously known. Although it is not at all obvious how to extend the definition of Newtonian capacity to a manifold, in some cases, as we have demonstrated above, it may be sufficient to define sets of zero or positive capacity. Finally I believe that capacity can play an importat role in other problems of integral geometry, particularly when terms to be integrated have singularities.

\section{BIBLIOGRAPHY}

1. R. Bott and S. S. Chern, Hermitian vector bundles and the equidistribution of the zeros of their holomorphic sections, Acta Math. 114 (1967), 71-112.

2. Lennart Carleson, Selected problems on exceptional sets, Van Nostrand, Princeton, N. J., 1967.

3. J. Carlson and P. Griffiths, The order functions for entire holomorphic mappings, Value Distribution Theory (edited by R. Kujala and A. Vitter, III), Dekker, New York, 1974.

4. J. Carlson, A moving lemma for the transcendental Bezout problem, Ann. of Math. (2) 130 (1976), 305-330.

5. M. Green, Holomorphic maps to algebraic varieties, Amer. J. Math. 97 (1975), 317-322.

6. P. Griffiths and J. King, Nevanlinna theory and holomorphic mappings between algebraic varieties, Acta Math. 130 (1973), 145-220.

7. J. Hirschfelder, First main theorem of value distribution, Invent. Math. 8 (1969), 1-33.

8. R. Molzon, Sets omitted by equidimensional holomorphic mappings, Amer. J. Math. 101 (1979), $1271-1283$.

9. W. Stoll, Invariant forms of Grassmann manifolds, Ann. of Math. Studies, no. 89, Princeton Univ. Press, Princeton, N. J., 1977.

10. H. Wu, Remarks on the first main theorem in equidistribution theory. III, J. Differential Geom. 3 (1968), 83-94.

11. Mappings of Riemann surfaces (Nevanlinna theory), Proc. Sympos. in Pure Math., vol. 11, Amer. Math. Soc., Providence, R. I., 1968, pp. 480-532.

DePARTMENT OF MATHEMATICS, UNIVERSITY OF KeNTUCKY, LEXINGTON, KeNTUCKy 40506

Current address: SFB 40, Theoretische Math., Universität Bonn, Beringstrasse 4, 5300 Bonn 1, BRD 